\title{
Correction to: Stiffness Control of Soft Robotic Manipulator for Minimally Invasive Surgery (MIS) Using Scale Jamming
}

S.M.Hadi Sadati, Yohan Noh, S. Elnaz Naghibi, Kaspar Althoefer, and Thrishantha Nanayakkara

Correction to:

Chapter "Stiffness Control of Soft Robotic Manipulator for Minimally Invasive Surgery (MIS) Using Scale Jamming" in: H. Liu et al. (Eds.): Intelligent Robotics and Applications, LNAI 9246, https://doi.org/10.1007/978-3-319-22873-0_13

The book was inadvertently published with an incorrect version of an author's name in Chapter 13 as "Althoefer Kaspar" whereas it has been updated as "Kaspar Althoefer". 